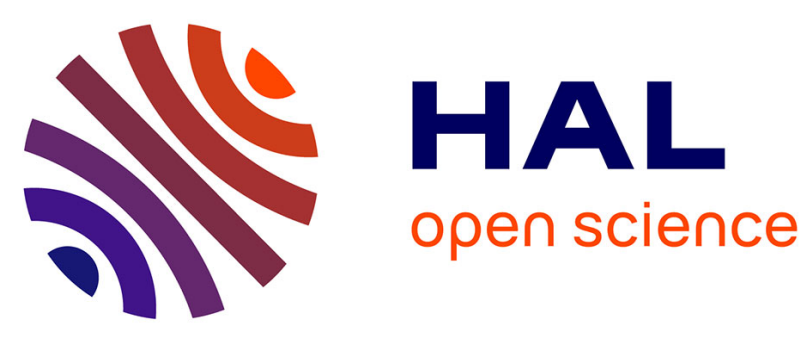

\title{
Bubble-induced turbulence
}

Frédéric Risso

\section{To cite this version:}

Frédéric Risso. Bubble-induced turbulence. Turbulent Cascades II, Springer, pp.189-196, 2019, Proceedings of the Euromech-ERCOFTAC Colloquium 589, 10.1007/978-3-030-12547-9_20 . hal02342841

\section{HAL Id: hal-02342841 \\ https://hal.science/hal-02342841}

Submitted on 1 Nov 2019

HAL is a multi-disciplinary open access archive for the deposit and dissemination of scientific research documents, whether they are published or not. The documents may come from teaching and research institutions in France or abroad, or from public or private research centers.
L'archive ouverte pluridisciplinaire HAL, est destinée au dépôt et à la diffusion de documents scientifiques de niveau recherche, publiés ou non, émanant des établissements d'enseignement et de recherche français ou étrangers, des laboratoires publics ou privés. 


\section{Bubble-induced turbulence}

Frédéric Risso

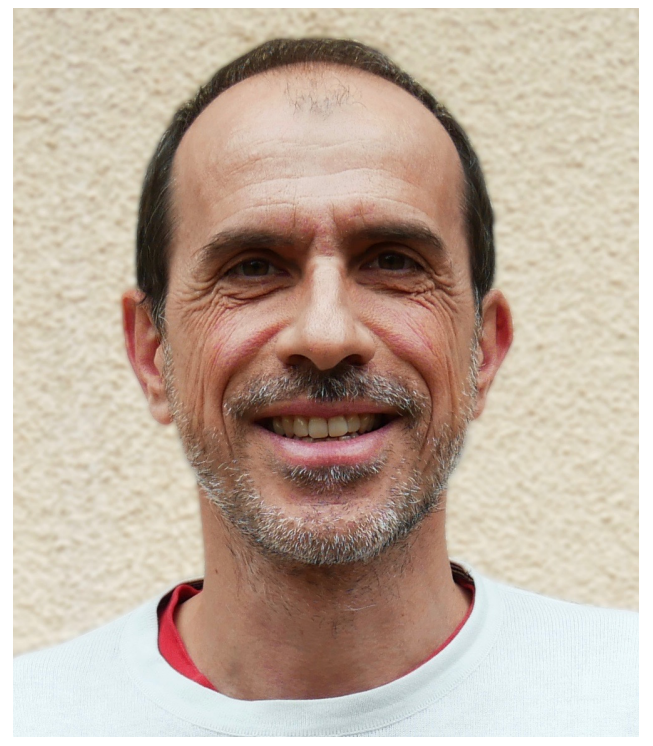

Abstract A homogenous swarm of bubbles rising through a liquid generates anisotropic homogeneous random velocity fluctuations. The statistical properties of bubble-induced fluctuations differ from the classical shear-induced turbulence. The probability density functions are non Gaussian and show a succession of exponential evolutions. The power spectral densities exhibit a $k^{-3}$ subrange for wavelengths around the bubble size. The understanding of these properties requires to consider

Frédéric Risso

Institut de Mcanique des Fluides de Toulouse, IMFT, Université de Toulouse, CNRS - Toulouse, FRANCE, e-mail: frisso.imft.fr 
that bubble-induced agitation involves two contributions of a different nature. The first one is not related to any flow instability and results from the anisotropic flow disturbances generated near the bubbles, principally in the vertical direction. The second one is the almost isotropic turbulence induced by the instability of the flow through a population of bubbles, which turns out to be the main cause of horizontal fluctuations. Even if the two contributions are coupled, only the second one deserves to be called bubble-induced turbulence.

\section{Agitation in a homogeneous bubble column}
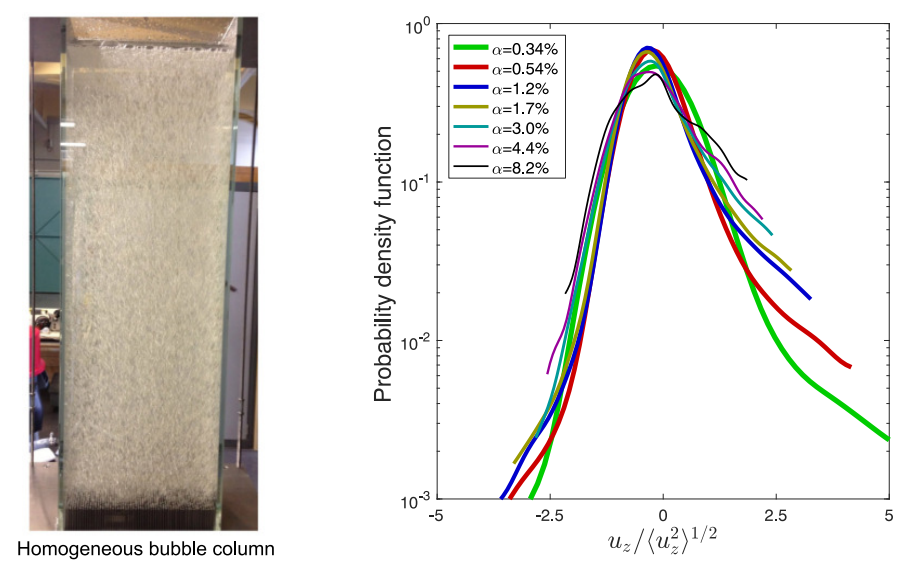

Fig. 1 Homogeneous bubble column. To the right: picture. To the left: probability density function of the vertical liquid velocity fluctuations for various gas volume fractions $\alpha$ from [4].

Bubbles rising through a liquid otherwise at rest generate random fluctuations. When the bubbles are uniformly distributed over space, the agitation of the liquid is homogeneous. Here, we consider situations where the Reynolds number $R e=$ $\langle V\rangle d / v$ (based on bubble average velocity $\langle V\rangle$, bubble diameter $d$ and kinematic viscosity of the liquid) is larger than 200. Since the pioneering work of Lance \& Bataille [2], many authors have investigated homogeneous bubble columns (left side of fig. 1), in which the average liquid velocity is everywhere zero. These works have been extensively reviewed in a recent state-of-the-art paper [8] and we recall here the salient features of bubble-induced agitation.

The variance of the fluctuations $\left\langle\mathbf{u}^{2}\right\rangle$ is roughly proportional to the product of the gaz volume fraction $\alpha$ and the square of the average bubble velocity. However, the fluctuations are anisotropic since the variance of the vertical velocity $\left\langle u_{z}^{2}\right\rangle$ is larger than that of the horizontal velocity $\left\langle u_{x}^{2}\right\rangle$. The probability density functions of both the horizontal and the vertical velocity fluctuations (PDFs) are non Gaussian. 
Moreover, the vertical PDFs are asymmetric with a long exponential tail for positive (upward) fluctuations, which becomes more and more important as $\alpha$ increases (right side of fig. 1).

The spectrum of the velocity fluctuations shows a subrange where the power density evolves as the power -3 of the wavenumber $k$. As shown in figure 2 , the $k^{-3}$ subrange is observed for wavelengths, $\lambda=2 \pi / k$, around the bubble diameter. Surprisingly, when normalised by the variance of the fluctuations, the spectra measured for various values of $\alpha$ collapse onto a single curve. This means that the integral length scale $\Lambda$ of the velocity fluctuations is independent of the gas volume fraction. The value of $\Lambda$ is related to the length of the wake that develops behind each bubble. The wake of a bubble that belongs to a swarm of rising bubbles is considerably shorter than the wake of an isolated rising bubble because of the interactions with neighbouring wakes. It decreases exponentially with the distance to the bubble on a length scale $L_{w} \approx 5 d$, which is observed to be independent to the gas volume fraction provided $\alpha \gtrsim 0.5 \%$.

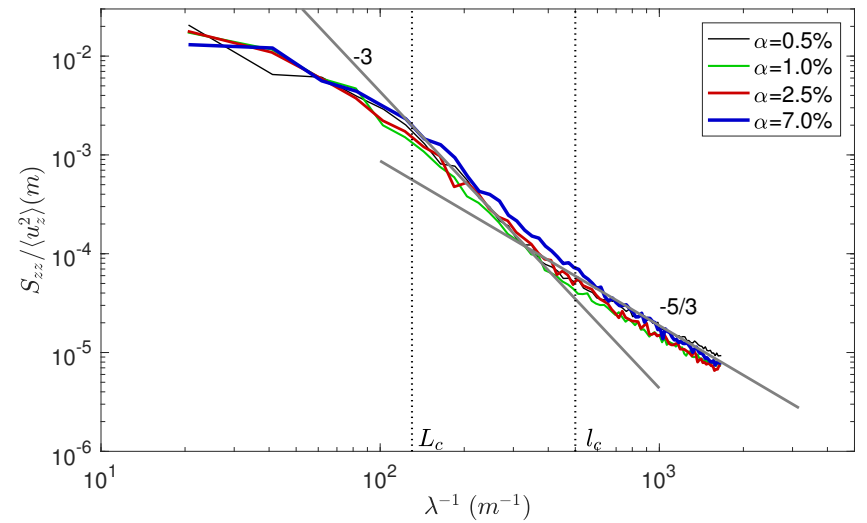

Fig. 2 Power spectra of liquid vertical velocity fluctuations measured in a bubble column by [4] $(d=2.5 \mathrm{~mm}, \alpha=1.7 \%, R e=760)$. The wavelengths limiting the $k^{-3}$ subrange are $l_{c}=2 \mathrm{~mm}$ and $L_{c}=7.7 \mathrm{~mm}$

\section{Distinction between turbulent fluctuations and average bubble disturbances}

The temporal velocity fluctuations which are measured in a given point located within a swarm of rising bubbles combine two contributions of a different nature. The first contribution corresponds to the average disturbance generated in the vicinity of each bubble. Even if the flow around each bubble would be steady in a frame 
that follows the bubble, temporal fluctuations would be observed in the laboratory frame as the bubble passes close to the measurement point. Since the bubbles are randomly distributed over space, random temporal fluctuations would be measured. It is clear that these fluctuations are not related to any kind of flow instability and must not be confused with turbulence. The second contribution is the real turbulence that develops provided the Reynolds number is large enough.
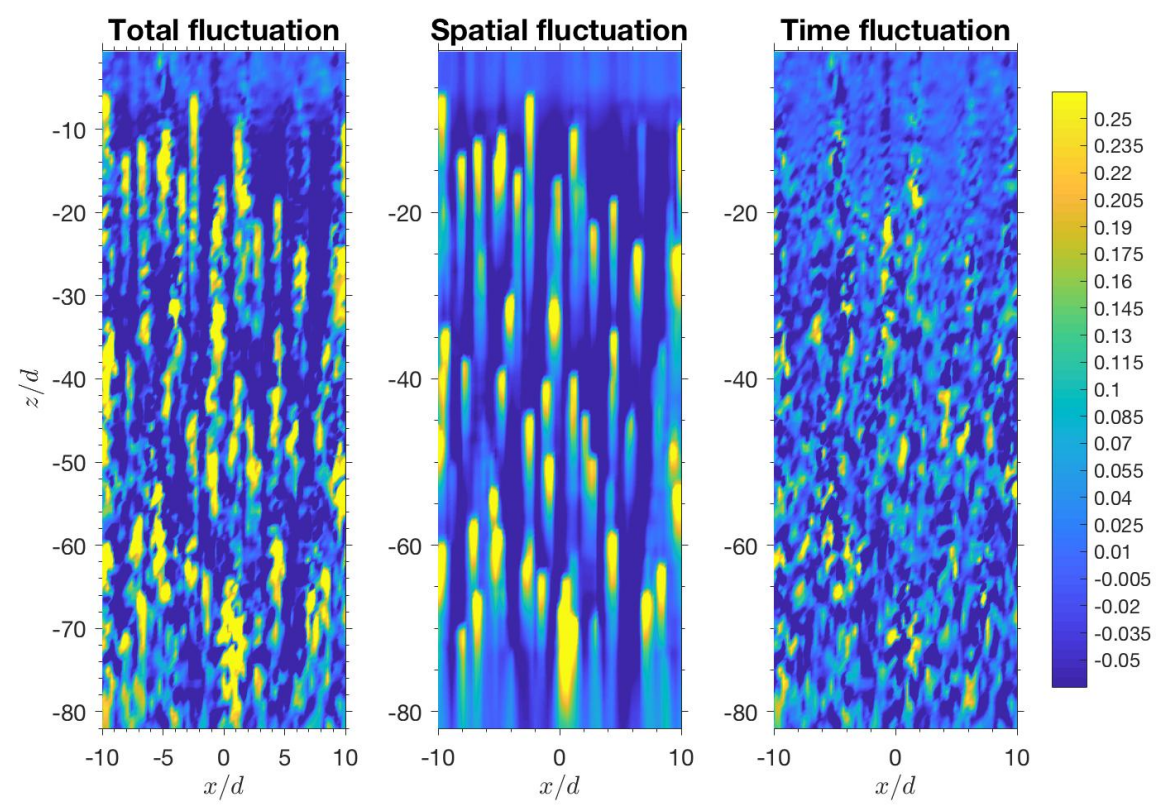

Fig. 3 Vertical velocity in a vertical plane computed by large-eddy simulation from [3]. Decomposition of velocity fluctuations by eq. 1 : (a) total fluctuation $u_{z}(\mathbf{x}, t)$; (b) spatial fluctuation $\bar{u}_{z}(\mathbf{x})$; (c) time fluctuation $u_{z}^{\prime}(\mathbf{x}, t)$.

It is easy to make the distinction between these two kinds of fluctuations in the idealized situation where the bubbles are not moving relatively to each other [5]. In this case, we can consider the liquid velocity in the frame where all the bubbles are at rest. Let us denote spatial averaging by angle brackets and time averaging by an overbar. After having removed the uniform average velocity, the liquid velocity $\mathbf{u}(\mathbf{x}, t)$ can be decomposed as

$$
\mathbf{u}(\mathbf{x}, t)=\overline{\mathbf{u}}(\mathbf{x})+\mathbf{u}^{\prime}(\mathbf{x}, t) .
$$

The spatial fluctuation $\overline{\mathbf{u}}(\mathbf{x})$ only depends on the spatial coordinate $\mathbf{x}$ and described the contribution of the individual bubble disturbances. The time fluctuation $\mathbf{u}^{\prime}(\mathbf{x}, t)$ depends on both the time $t$ and the location $\mathbf{x}$ and accounts for the turbulence. The 
variance of the total fluctuation $\mathbf{u}(\mathbf{x}, t)$ is the sum of the variances of these two contributions:

$$
\left\langle\mathbf{u}^{2}\right\rangle=\left\langle\overline{\mathbf{u}}^{2}\right\rangle+\left\langle\mathbf{u}^{2}\right\rangle .
$$

Such a decomposition has been applied to results of an experimental investigation of the flow through a random array of fixed spheres [1] and to results of largeeddy simulations (LES) of the flow through a fixed swarm of bubbles [3]. Figure 3 presents the fields of total, spatial and time vertical velocity fluctuations obtained by LES. The spatial fluctuations clearly exhibits the bubble wakes whereas the time fluctuations are almost the same everywhere and therefore not strongly correlated to the bubble locations. From these investigations of flows through random array of obstacles, the following conclusions are obtained. The fluctuations due to individual bubble disturbances are strongly anisotropic and constitute the dominant contributions to the total fluctuations in the vertical direction. The turbulent fluctuations are almost isotropic, predominant in the horizontal direction, and their PDFs are exponential. Regarding the spectrum, the bubble disturbances are dominant at larger scales while the turbulence is preponderant at smaller scales. However, both contributions show a $k^{-3}$ spectral subrange.

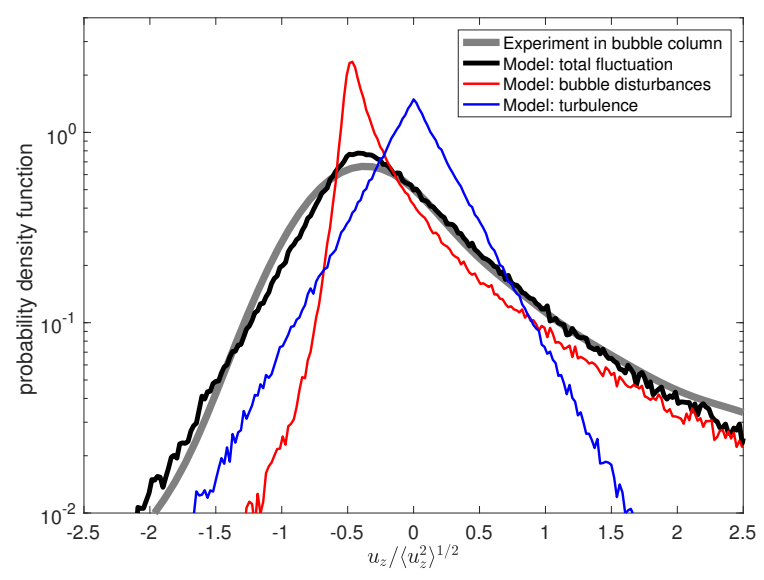

Fig. 4 Probability density function of vertical liquid velocity fluctuations: Comparison between experimental results measured in a bubble column by [4] $(\alpha=1.7 \%, \operatorname{Re}=760)$ and model by [7].

Having regard to the results obtained in idealized flows, a model for the fluctuations in a real bubble swarm was proposed by [7]; it is shortly summarized below.

The bubble disturbances are described by considering that the flow around a given bubble is the sum of the potential flow around the bubble and an exponential decaying wake, which is characterized by a length scale $L_{w}$. The locations of a large number of bubbles are randomly chosen independently of each other. The disturbances generated by all these bubbles at a given point are linearly superimposed. 
A sample of velocity fluctuation is thus obtained. This operation is repeated a great number of times in order to obtain a statistically significant ensemble of samples, from which a PDF is computed. Figure 4 compares the model PDF of the vertical fluctuations due to bubble disturbances (red curve) to an experimental PDF (grey curve) measured in a homogeneous bubble column. Note that the value of $L_{w}$ has been adjusted to fit the experimental tail of positive fluctuations. We see that if the model is able to reproduce well the tail of positive fluctuations, which is dominated by the wakes, it fails to reproduce the negative fluctuations.

Assuming that turbulence is isotropic, the three components of the turbulent fluctuations are model by the same exponential PDF:

$$
f_{t}\left(u^{\prime}\right)=\frac{\sqrt{2}}{2 \sigma_{t}} \exp \left(-\frac{\sqrt{2}\left|u^{\prime}\right|}{\sigma_{t}}\right),
$$

where the value of $\sigma_{t}$ is chosen to fit the central part of the experimental PDF of the horizontal fluctuations (not shown here). The blue curve in fig. 4 shows the model PDF of the vertical fluctuations due to bubble-induced turbulence.

The model for the total fluctuations is obtained by assuming that the turbulent fluctuations are independent of the bubble locations, which implies that fluctuations induced by bubble disturbances and turbulence are statistically independent. A sample of the total fluctuations is thus obtained as the sum of a sample of each contribution. The model PDF for the total vertical fluctuations is represented by a black curve in fig. 4. It matches well the experimental results.

This model has been satisfactorily compared to experimental results obtained for various gas volume fractions and several bubble diameters. In agreement with results obtained from investigations of flows through arrays of fixed obstacles, this confirms that the turbulent contribution to bubble-induced agitation in a real bubble column is almost isotropic and well described by an exponential PDF.

\section{Discussion of the spectrum of the bubble-induced turbulence}

The bubble-induced turbulence has two important features that may help to shed light on its spectral behaviour: (1) It exhibits a $k^{-3}$ power law within a range of scales around the bubble size where the motions of the bubble supply energy to the liquid; (2) The wavenumber spectrum is invariant with $\alpha$ when normalized by the variance $\left\langle u^{\prime 2}\right\rangle$ and the integral length scale $\Lambda$ of the fluctuations.

Let us start analysing property (1) by considering the energy balance in the spectral domain. In a statistically steady and homogeneous state, the power spectral density $E$ of the turbulent fluctuations $u^{\prime}$ is related to the energy production $\Pi$ and to the energy transfer $T$ between the wavenumbers by

$$
2 v k^{2} E=T+\Pi .
$$


Lance \& Bataille [2] proposed that the turbulent fluctuations were localized within the bubble wakes where there were both produced and dissipated. Assuming that the production only depends on the dissipation rate $\varepsilon$ and on the wavenumber $k$, dimensional considerations leads to $\Pi \propto \varepsilon k^{-1}$. Inserting this expression in eq. 4 and neglecting $T$, they obtained a spectrum in $k^{-3}$ :

$$
E \propto \frac{\varepsilon k^{-3}}{2 v} .
$$

Owing to the results of LES in which the scales smaller than the wakes are not resolved [3] and the fact that a $k^{-3}$ subrange has been observed in experiments for scales larger than $d$ [4], the assumption that turbulence is localized within the wakes is invalided. However, the assumption that $\Pi$ and $T$ depend only upon $\varepsilon$ and $k$ is a less restrictive assumption that still leads to eq. 5 .

Then, property (2) implies the following relation for the $k^{-3}$ subrange:

$$
\frac{E}{\left\langle u^{\prime 2}\right\rangle \Lambda} \propto(\Lambda k)^{-3} \text {. }
$$

Equating eqs. 5 and 6 yields

$$
\varepsilon \propto v \frac{\left\langle u^{2}\right\rangle}{\Lambda^{2}},
$$

where $\Lambda$ appears as sort of Taylor scale for the $k^{-3}$ subrange. Taking into account that $\varepsilon=\alpha g\langle V\rangle$ and $\left\langle u^{2}\right\rangle \propto \alpha\langle V\rangle^{2}$ finally leads to the following scaling for $\Lambda$ :

$$
\Lambda \propto \sqrt{\frac{v\langle V\rangle}{g}} \propto \frac{d}{\sqrt{C_{d} R e}} .
$$

The interest of this relation is that it can be tested in experiments or simulations to assess the validity of the assumptions leading to eq. 5. For the experiments of [4], taking the values of $R e$ and $C_{d}$ corresponding to an isolated bubble, eq. 8 leads to the same value of $\Lambda$ for the three investigated bubble diameters, which is in agreement with the observations. In fig. 5, the various scalings for $\Lambda$ have been compared to the results obtained by [1] in an random array of fixed spheres for various $R e$ by estimating $C_{d}$ by the Schiller and Naumann relation for an isolated solid sphere. It turns out that eq. 8 gives the correct evolution of $\Lambda$. Comparisons with other investigations covering a broader range of parameters are nevertheless required to reach a definitive conclusion regarding the validity of eqs. 5 and 8 .

\section{Conclusion}

Bubble-induced agitation combines two contributions: the fluctuations generated by the disturbances localized around each bubble and real bubble-induced turbu- 


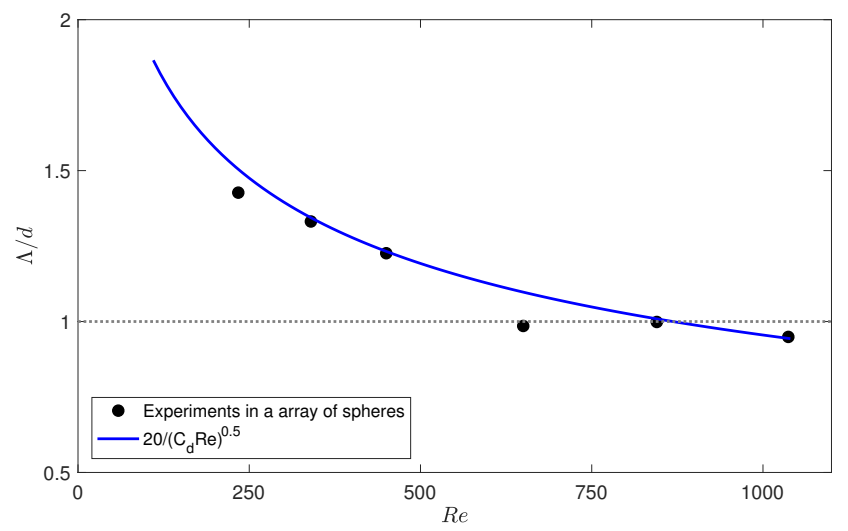

Fig. 5 Evolution of the Eulerian integral length scale as a function of the Reynolds number in an experimental random array of spheres by $[1](\alpha=2 \%)$.

lence. The major statistical properties of both contributions have been determined by many authors and are now well known. On the one hand, the role of the localized bubble disturbances is now well understood since their PDFs have been satisfactorily described by considering the summation of individual disturbances [7] and their spectral behaviour reproduced by considering a succession of random bursts [6]. On the other hand, the exponential PDFs and the $k^{-3}$ spectral subrange of the bubbleinduced turbulence are still not understood. They are probably the consequences of the instability of the flow through a random array of obstacles.

\section{References}

1. Amoura Z., Besnaci C., Risso, F. and Roig V.: The flow through a random array of spheres as a model of bubble-induced agitation. J. Fluid Mech. 823, 592-616 (2017)

2. Lance M. and Bataille J.: Turbulence in the liquid-phase of a uniform bubbly air water-flow. J. Fluid Mech. 222, 95-118 (1991)

3. Riboux G., Legendre D. and Risso F.: A model of bubble-induced turbulence based on largescale wake interactions. J. Fluid Mech. 719, 362-387 (2013)

4. Riboux G., Risso F. and Legendre D.: Experimental characterization of the agitation generated by bubbles rising at high Reynolds number. J. Fluid Mech. 643, 509-539 (2010)

5. Risso F., Roig V., Amoura Z., Riboux G. and Billet A.-M.: Wake attenuation in large Reynolds number dispersed two-phase flows. Phil. Trans. R. Soc. A 366, 2177-2190 (2008)

6. Risso F.: Theoretical model for $k^{-3}$ spectra in dispersed multiphase flows. Phys Fluids. 23, 011701 (2011)

7. Risso F.: Physical interpretation of probability density functions of bubble-induced agitation. J. Fluid Mech. 809, 240-263 (2016)

8. Risso F.: Agitation, mixing, and transfers induced by bubbles. Annu Rev. Fluid Mech. 50, 25-48 (2018) 